\title{
Influence of sonication and in vitro evaluation of nifedipine self-nanoemulsifying drug delivery system
}

\author{
Ahmad M Eid ${ }^{1 *}$, Nagib A Elmarzugi ${ }^{\circledR 2}$, Nidal A Jaradat ${ }^{1}$ \\ ${ }^{1}$ Department of Pharmacy, Faculty of Medicine and Health Sciences, An-Najah National University, Nablus, Palestine, \\ ${ }^{2}$ Department of Industrial Pharmacy, Faculty of Pharmacy, Tripoli University \& National Nanotechnology Project, \\ Biotechnology Research Center, Tripoli, Libya
}

\begin{abstract}
In order to develop a self-nanoemulsifying system, three components, olive oil, Tween 80, and Capmul, were used to construct a ternary phase diagram that helped to find the optimum formulation, which was loaded with nifedipine. The effect of sonication on drug loading was also evaluated. After that, measurement of the droplet size, size distribution, zeta potential, and scanning electron microscopy were conducted for evaluation and characterisation of the formulations. The phase diagram of four formulations showed nanosizes below $200 \mathrm{~nm}$; however, only one was selected to be loaded with nifedipine. The selected formulation had the lowest droplet size of $98 \mathrm{~nm}$ and size distribution 0.192 , and was composed of $48 \%$ Tween $80,32 \%$ Capmul, and $20 \%$ olive oil. The nifedipine self-nanoemulsifying drug delivery system (SNEDDS) showed a significant change in the particle size $(97 \mathrm{~nm})$ and size distribution $(0.257)$ after sonication. Its zeta potential was $-32.3 \mathrm{mV}$ indicating good stability. The SEM photographs of nifedipine showed particles with spherical shape and smooth surface. Finally, a self-nanoemulsifying formulation containing nifedipine, loaded in olive oil, was successfully prepared by mixing the oil with various types of surfactants and co-surfactants. A significant nifedipine self-nanoemulsifying system was developed and significantly improved accordingly.
\end{abstract}

Keywords: Nifedipine. Self-nanoemulsifying drug delivery system. Sonication. SEM.

\section{INTRODUCTION}

The pharmaceutical industry is currently facing difficulties in the development of new drugs for oral delivery, especially those with the poor water solubility frequently associated with low bioavailability. Furthermore, oral dosage forms must be able to offer the greatest degree of patient compliance, be easy to handle, and have a low cost per unit dose (Avdeef, 2012). Recently, the usage of medicinal nanotechnology formulations has grown in the pharmaceutical industry (Parveen, Misra, Sahoo, 2012). They are considered as a solution for many problems facing the pharmaceutical industry during the research and development of new drug (Kumar, 2010). In addition, the pharmaceutical industry has used nanotechnology in enhancing drug formulation,

\footnotetext{
*Correspondence: A. M Eid. Pharmaceutical Chemistry and Technology Division, Department of Pharmacy, Faculty of Medicine and Health Sciences, An-Najah National University, Nablus. Palestine. P.O. Box 7. E-mail: ahmadeid@najah.edu
}

drug stability, and drug targeting to specific disease sites (Nikalje, 2015).

Maximising the therapeutic index of the drug delivery system and reducing its side effects has become the main concern for the pharmaceutical industry (Keohane et al., 2016; Shakeel et al., 2013). Furthermore, it can improve the ease of administration, thus indirectly improving patient compliance (Cerpnjak et al., 2015). Many researchers have presented that a self-nanoemulsifying drug delivery system (SNEDDS) is one of the best methods available to improve the oral bioavailability of poorly soluble drugs (Badran et al., 2014; Balakrishnan et al., 2009; Balakumar, Raghavan, Abdu, 2013; Hintzen et al., 2014). A study conducted by Vanani, Moezi, and Heli (2017) showed a significant increment in the absorption of curcumin in a self-nanoemulsifying system, leading to an enhancement in its bioavailability (Vanani, Moezi, Heli, 2017). Particle sizes less than $200 \mathrm{~nm}$ are usually produced, which can improve the chemical and/or physical stability profile in addition to providing long lasting storage (Memvanga, 
Coco, Préat, 2013; Swain, Patra, Rao, 2016).

ASNEDDS is an anhydrous homogeneous liquid drug delivery system consisting of oil, surfactant, co-emulsifier or solubiliser, and drug. SNEDDS upon dilution with water under gentle stirring will self-nanoemulsify into oil-in-water nanoemulsion spontaneously with about $200 \mathrm{~nm}$ or less in average size (Eid et al., 2014a; Kuentz, 2012; Mohsin et al., 2016). Usually, SNEDDS can readily spread in the gastrointestinal tract, which is due to the digestive motility of the intestine and the stomach that provides the agitation necessary for the self-emulsification process (Czajkowska-Kośnik et al., 2015; Mahmoud, Shukr, Bendas, 2014). The selection of SNEDDS components depends on the solubilisation capacity and physicochemical and physiological properties of the drug (Dash et al., 2015; Eid, El-Enshasy, 2014a; Kale, Patravale, 2008). Therefore, hydrophobic drugs are one of the candidates for SNEDDS. This system helps to improve drug oral bioavailability by several mechanisms; usually it is governed by the size of nanoparticles and the enhancement of dissolution (Abdalla, Klein, Mäder, 2008; Arslan, Tirnaksiz, 2013; Kohli et al., 2010).

Nifedipine is a long- and short-acting 1,4-dihydropyridine calcium channel blocking agent. It is widely used in the management of hypertension and in the treatment of angina pectoris (Mancia et al., 2003; Richard, 2005). Nifedipine is a lipophilic drug and, thus, has poor water solubility but is soluble in ethanol. Moreover, it is very sensitive to light. Its poor aqueous solubility is considered the major problem associated with formulations of nifedipine. Its solubility and $\mathrm{pH}$ range are 5-6 $\mu \mathrm{g} / \mathrm{mL}$ and $4-13$, respectively; those factors may cause it to exhibit poor pharmacokinetic properties, and also low and irregular bioavailability in humans (Hecq et al., 2005).

This study was conducted to develop and optimise formulations containing surfactants reported to be bioenhancers, which can help in the development of a nifedipine self-nanoemulsifying drug delivery system, and to study the effect of sonication on the formulation of nifedipine SNEDDS. Studying SNEDDS can provide important tools for the development of a drug delivery system that could contribute to better pharmaceutical products in the future.

\section{MATERIAL AND METHODS}

\section{Material}

Nifedipine (Merck, Germany), Tween 80, Capmul MCM (Natural Wellbeing, Malaysia), and olive oil (Olitalia, Italy).

\section{Pseudo-ternary phase diagram study}

A pseudo-ternary phase diagram was constructed by employing a series of formulations containing oil, surfactant, and co-surfactant. The mixtures of olive oil, Tween 80, and Capmul were weighed out with an analytical balance (Metler Tolledo). Then, the samples were put in vortex for 2 minutes in order to mix the components of the formulation. A sample of $250 \mu \mathrm{L}$ from the formulation was self-emulsified in $50 \mathrm{~mL}$ of distilled water for visual observation and to evaluate its droplet size and size distribution to find the region of emulsification.

After observation of the droplet size was completed, the emulsion region was identified in the phase diagram for nanoemulsion (NE) region. It was identified due to transparent and fine particles. Meanwhile, the macroemulsion (ME) region was identified and appeared to have more whitening and isotropic solutions that might involve micelles, and coarse emulsion (CE) was the region of visibly cloudy dispersions observable even by visual observation. Some of the non-emulsified phases are not shown in the phase diagrams since they are beyond the scope of the present work. The optimum self-nanoemulsifying formulations were selected based on their droplets size (below $200 \mathrm{~nm}$ ) and size distribution (below 0.3) (Eid et al., 2014b); those formulations were loaded with nifedipine to check whether the particle size of the formulation reached the nanosize.

\section{Nifedipine loading into a self-nanoemulsifying system}

Nifedipine $(10 \mathrm{mg})$ was incorporated into the selected self-nanoemulsifying formulation. Nifedipine was loaded in the selected formulation by mixing it with olive oil until it dissolved completely in the oil and then mixing it with the surfactants (Tween 80 and Capmul) in an Eppendorf tube. The effect of sonication was evaluated by sonicating the formulation for 30 minutes in a $48^{\circ} \mathrm{C}$ water bath to facilitate the solubilisation and vortex mixing. Each formulation was vortexed again for 5 minutes at room temperature at $25^{\circ} \mathrm{C}$. Then, the nanoemulsion produced was analysed using a Malvern Zetasizer Nano ZS (Malvern Instruments, UK) and scanning electron microscopy (SEM) (Gupta et al., 2016).

\section{Visual observation}

Approximately $250 \mu \mathrm{L}$ of the formulation was gently agitated using a glass rod after it was introduced 
to $50 \mathrm{~mL}$ of distilled water in a $100 \mathrm{~mL}$ beaker. The progress of the emulsion droplets and also the tendency to spontaneously emulsify were observed. When the mixture readily formed a fine milky emulsion with droplets that spread easily in water, the emulsion was judged as "good" and it was judged "moderate" when a droplet was not quickly emulsified and produced milky emulsion. Lastly, it was judged "bad" when there was no or poor emulsion with immediate coalescence of oils droplets, especially when gentle agitation was stopped (Schuh, Bruxel, Teixeira, 2014). In this study, the ease of emulsion formation and dispersion in terms of its ability to spread and mix with water were observed. The time taken for the emulsion to be emulsified was expressed in terms of emulsification time.

\section{Particle size and zeta potential analysis}

Fifty millilitres of distilled water were placed in a $100 \mathrm{~mL}$ beaker, then $250 \mu \mathrm{L}$ of an oil/surfactants mixture were introduced and mixed using a glass rod to induce gentle agitation. For the screening study and construction of the ternary phase diagram, the resultant emulsion was tested for its droplet size and size distributions using a Malvern Mastersizer 2000MU laser diffraction particle analyser. Zeta potential was also performed by using Malvern Zetasizer Nano ZS for the optimum formulation (Da Costa et al., 2014).

\section{Scanning electron microscopy}

SEM was used to analyse the morphology of the nanoemulsion particles. The formulation was prepared as described before and then $250 \mu \mathrm{L}$ of the formulation were self-emulsified in $50 \mathrm{~mL}$ of distilled water in a $100 \mathrm{~mL}$ beaker under gentle agitation. The sample was centrifuged using a Sartorius Sigma Centrifuge (Sigma Instrument, Germany) at 10,000 rpm for 5 minutes to form pellets. Then, $2 \%$ osmium tetroxide was mixed into the pellet for 1 hour before imaging (Majeed et al., 2015).

\section{Statistical analysis}

For statistical analysis, the Statistical Package for Social Science (SPSS) version 16 and Microsoft Excel 2010 were used to analyse the obtained results. The results were expressed as mean \pm standard deviation (SD). Statistical significance was considered when the $p$ value was $<0.05$.

\section{RESULTS AND DISCUSSION}

\section{Pseudo-ternary phase diagram}

The pseudo-ternary phase diagram was constructed in order to find the self-nanoemulsifying region as shown in Figure 1. It was observed that there were different area divisions in the diagram. The NE area was the area that gave nanoemulsion droplets that were below than $1 \mu \mathrm{m}$. Meanwhile, the ME area was the area that gave macroemulsion droplets ranging in size from larger than $1 \mu \mathrm{m}$ to less than $20 \mu \mathrm{m}$. The $\mathrm{CE}$ region was the area of the coarse-nanoemulsion formulations.

For this study, olive oil was used to mix with Tween 80 and Capmul MCM before loading with nifedipine to construct the pseudo-ternary phase diagram. More accurate results are usually found with a combination of different types of surfactants compared to the results of using only a single surfactant. It is critical to choose the surfactants with the right blend of high and low hydrophile lipophile balance (HLB) when developing a selfemulsifying formula to produce a successful formulation of a microemulsion. Surfactants with high HLB values have rapid self-emulsification and excellent spreading properties (Pouton, 2000). On the other hand, low HLB value surfactants exhibit poor spreading characteristics, long emulsion times, and poor self-emulsifying properties. The formation of a self-nanoemulsifying system is critical because it depends on the selection of surfactant mixtures. Usually there is a specific HLB value for each surfactant and oil. The selection of the right HLB for a surfactant or blend of surfactants, which match the oil HLB, will lead to

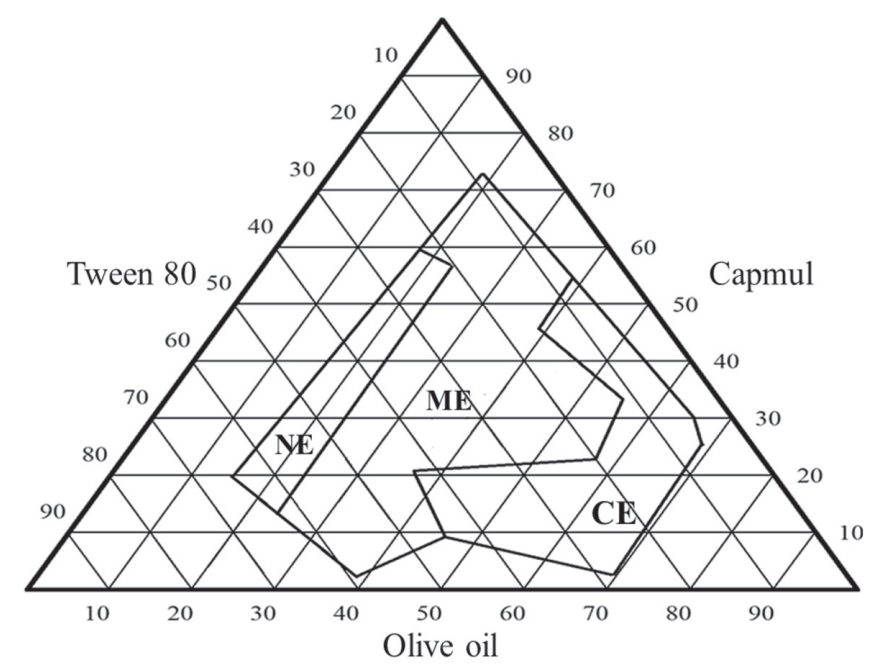

FIGURE 1 - Pseudo-ternary diagram for all the formulations. $\mathrm{NE}$ : nanoemulsion, $\mathrm{ME}$ : macroemulsion and $\mathrm{CE}$ : course emulsion. 
TABLE I - The selected formulations that have droplet sizes below $200 \mathrm{~nm}$

\begin{tabular}{cccccc}
\hline Formulation & Tween 80 (\%) & Capmul (\%) & Olive Oil (\%) & Droplet Size $\mathbf{n m} \pm$ SD & Size Distribution \pm SD \\
\hline 1 & 26 & 54 & 20 & $182 \pm 5.8$ & $0.446 \pm 0.016$ \\
2 & 32 & 48 & 20 & $149 \pm 5.5$ & $0.327 \pm 0.017$ \\
3 & 48 & 32 & 20 & $98 \pm 3.8$ & $0.192 \pm 0.008$ \\
4 & 64 & 16 & 20 & $193 \pm 1.5$ & $0.385 \pm 0.012$ \\
\hline
\end{tabular}

providing the lowest interface tension between the oil and water phases. In addition, a very stable system at a lower level is provided when the HLB of the oil and surfactant used in the preparation of a self-nanoemulsifying system are similar. Self-emulsifying systems are very sensitive to the ratio of oil/surfactant mixtures.

\section{Selected formulations}

The results of the selected formulations, which contain olive oil mixed with Tween 80 and Capmul MCM, are shown in Table I. Out of all the formulations used to construct the ternary phase diagram, only four formulations gave nanosizes below $200 \mathrm{~nm}$. The formulations with sizes below $200 \mathrm{~nm}$ were selected based on the criteria of the nanometric scale (20-200 nm) (Constantinides, Chaubal, Shorr, 2008; Eid, El-Enshasy, 2014b; Gutiérrez et al., 2008; Tadros et al., 2004).

Four formulations were found to have droplet sizes below $200 \mathrm{~nm}$ with uniformity between 0.192 and 0.446 (Figure 2). From the currently obtained results, formulation 3 gave the smallest droplet size of $98.0 \pm 3.8 \mathrm{~nm}$ with $0.192 \pm 0.008$ size distribution compared to other formulations. Therefore, formulation 3 was selected for further evaluations as it had the lowest droplet size and size distribution, which are usually attributed to the better surfactant and co-surfactant mixture. A smaller droplet size may lead to better drug absorption and bioavailability, while the narrow size distribution is an indication of more uniform emulsion with better stability (Nasr, Gardouh, Ghorab, 2016).

\section{Visual observation}

The visual observation of the selected formulations characterised the spreadability, time of emulsification, and appearance of each formulation as shown in Table II. All of the formulations had good spreadability with emulsification times between 55 to 26 seconds, and a transparent appearance.

The proportions of oil, surfactants, and co-surfactants in the mixture and the chemical structure of the oil will

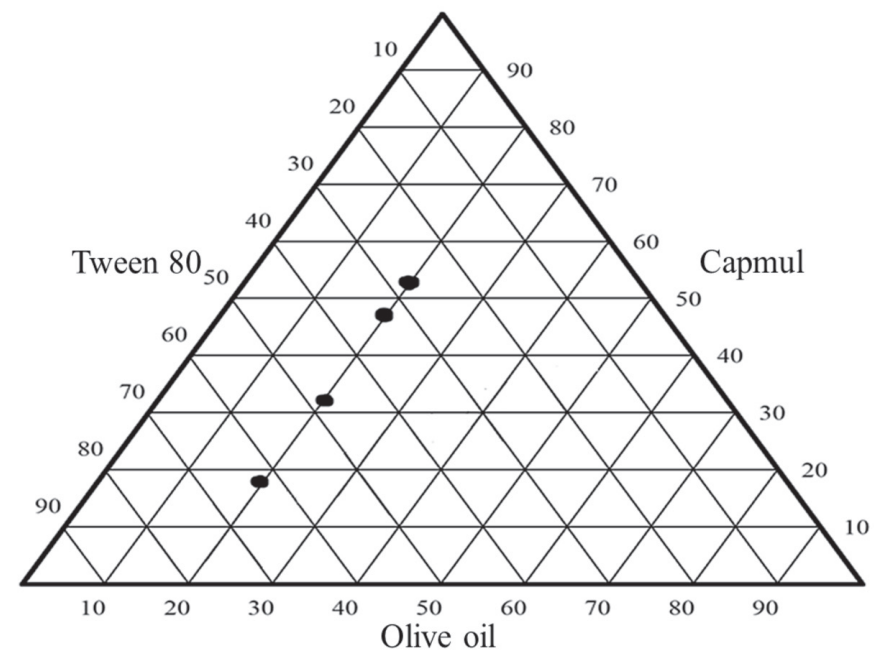

FIGURE 2 - The selected Self-nanoemulsifying formulations on the ternary phase diagram.

TABLE II - Visual observation of the self-nanoemulsifying formulations

\begin{tabular}{cccc}
\hline Formulation & Spreadability & Time (s) $\pm \mathbf{0 . 5}$ & $\begin{array}{c}\text { Emulsion } \\
\text { Appearance }\end{array}$ \\
\hline A & Easily Spread & 55 & Transparent \\
B & Easily Spread & 50 & Transparent \\
C & Easily Spread & 34 & Transparent \\
D & Easily Spread & 26 & Transparent \\
\hline
\end{tabular}

determine the emulsification times. This study revealed that the concentrations of surfactants and co-surfactants in the mixture had an inverse relationship with emulsification time. As the proportion of surfactant and co-surfactant increased, the self-emulsifying process became more difficult. The viscosity of the formulation usually would increase when the surfactant content increases. Hence, this led to formation of an emulsion with longer time of emulsification. Similar findings were also stated by Gao et al. (1998) who confirmed that an increase in the emulsification time would result from an increase in the surfactant and co-surfactant concentrations (Gao et al., 1998). Overall, all of the formulations had good self- 
TABLE III - Mean droplet size, size distribution, and zeta potential of formulations X and Y

\begin{tabular}{cccc}
\hline Formulation & Droplet Size $\mathbf{n m} \pm$ SD & Size Distribution \pm SD & Zeta Potential $(\mathbf{m V}) \pm$ SD \\
\hline $\mathrm{X}$ & $594 \pm 5.2$ & $1.1 \pm 0.12$ & $-19.4 \pm 2.4$ \\
$\mathrm{Y}$ & $97 \pm 2.1$ & $0.257 \pm 0.017$ & $-32.3 \pm 1.5$ \\
\hline
\end{tabular}

emulsification properties with the ability to form a cloudy emulsion in less than 60 seconds and having good spreading characteristics (Craig et al., 1995).

\section{Analysis of nifedipine self-nanoemulsifying system before and after sonication}

Formulation 3 was noted to be the best formulation based on the results of the droplet size obtained from the Malvern Mastersizer with the lowest droplet size at $98 \mathrm{~nm}$ and size distribution of 0.192 . Therefore, it was selected to be loaded with nifedipine.

Formulation X represents the formulation loaded with nifedipine but without sonication, whereas formulation $\mathrm{Y}$ was sonicated for 20 minutes using an ultrasonic sonicator bath. Table III shows the results of the droplet size, size distribution, and zeta potential for both formulations $\mathrm{X}$ and $\mathrm{Y}$.

After selecting the optimum formulation, two formulations were loaded with nifedipine and vortexed for 5 minutes. Formulation $\mathrm{Y}$ was sonicated for 20 minutes while formulation $\mathrm{X}$ was not sonicated. The results showed that formulation Y presented a smaller mean particle size $(97.0 \pm 2.1 \mathrm{~nm})$ compared to formulation $\mathrm{X}$ $(594.0 \pm 5.2 \mathrm{~nm})$. Even though both of the formulations showed nanosize particles, formulation Y showed the smallest particle size, thus achieving our study objective. Furthermore, formulation Y showed a better size distribution of less than 0.3 , (which was $0.257 \pm 0.017$ ) compared to formulation $\mathrm{X}$ with a size distribution of $1.10 \pm 0.12$.

In addition, the drug particles in the formulations had different physicochemical characteristics, one of which was zeta potential. The stability of the formulation can be influenced by the charge of the substance. Zeta potential is the potential between the surface of a droplet and the dispersing liquid. It will vary according to the distance of the ion from the droplet surface. In general, emulsions are considered to be stable with negative or positive zeta potential of more than $30 \mathrm{mV}$ (Stolnik et al., 1994), i.e. a stable or unstable emulsion is generally considered at either +30 or $-30 \mathrm{mV}$. The zeta potential of formulation $\mathrm{Y}$ was lower than $-30 \mathrm{mV}$, which indicates that the formulation has better stability compared to X.
The current results are in accordance with Nekkanti et al. (2010) who prepared a solid self-microemulsifying drug delivery system for candesartan cilexetil and found its zeta potential values were below $-30 \mathrm{mV}$ (Nekkanti et al., 2010). Sonication is a highly complex system involving a variety of concomitant physicochemical interactions that can result in either cluster breakdown of the drug particle leading to reduction of the average particle size, as well as other effects including particle size distribution and drug solubility in the oil (Pradhan et al., 2016). Therefore, it is concluded that sonication can be indicative of more effectiveness in preparing a self-nanoemulsifying drug delivery system.

\section{Morphology of nifedipine particles}

Nifedipine particles in formulation $\mathrm{Y}$ were tested for their morphology using SEM, as it had lower droplet size and size distribution with better zeta potential compared to X. Figure 3 shows the SEM image of the self-nanoemulsifying formulation that was loaded with the nifedipine.

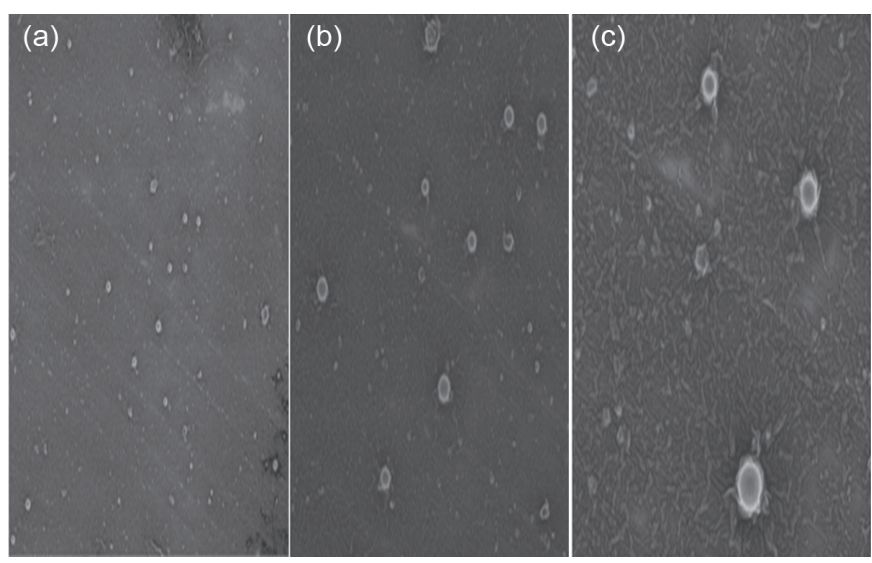

FIGURE 3 - Representative SEM photographs Nifedipine self-nanoemulsifying system (a) 1000_ magnification, (b) 2500_magnification and (c) 5000_magnification.

SEM is a technique to study the surface topography of a sample and a gives a 2-D image of the particles (Divsalar et al., 2012). SEM is considered a critical tool to acquire specific information about basic structural properties of colloidal drug delivery systems (Czajkowska-Kośnik, 
Szekalska, 2015). All nanoparticles showed a spherical shape with a smooth surface at higher magnifications. They had a few large particles among a large population of small particles. The larger particles may affect the long term stability of the nanoemulsion; thus, it is considered to be unsuitable to be used for intravenous applications (Klang et al., 2012). The solution to this problem is to increase the vortex time or increase the sonication time. SEM images obtained for the nifedipine selfnanoemulsifying system are shown in Figure 3. The SEM photographs show that the obtained particles had a spherical shape and were characterised by a smooth surface.

\section{CONCLUSION}

SNEDDS might be a promising approach for the rapid onset and the effective absorption for oral administration of nifedipine, thereby increasing the bioavailability of the drug. A self-nanoemulsifying formulation containing nifedipine, loaded in olive oil, was successfully prepared by mixing the oil with various types of surfactant and co-surfactants. In general, the self-nanoemulsifying formulations were significantly improved and had better self-nanoemulsifying properties for long term stability when it was sonicated.

\section{AUTHORS' CONTRIBUTIONS}

Ahmad Eid conceived, accomplished and designed the paper, Nagib and Nidal carried out the data obtaining and drafted this paper. All researchers collected the informants, revised, read and approved the final manuscript.

\section{ACKNOWLEDGEMENTS}

This study was not funded by any institution. Sincere thanks to all participants in the study are also acknowledged. I want to give my gratitude to Mrs. Cynthia Mizyed for her proofreading, which has profoundly improved the composition of this paper.

\section{CONFLICT OF INTEREST}

The authors declare no conflict of interest.

\section{REFERENCES}

Abdalla A, Klein S, Mäder K. A new self-emulsifying drug delivery system (SEDDS) for poorly soluble drugs: characterization, dissolution, in vitro digestion and incorporation into solid pellets. Eur J Pharm Sci. 2008;35(5):457-464.
Arslan SA, Tirnaksiz F. Self-emulsifying drug delivery systems. FABAD J Pharm Sci. 2013;38(1):55-64.

Avdeef A. Absorption and drug development: Solubility, permeability, and charge State. New Jersey: John Wiley \& Sons; 2012.

Badran MM, Taha EI, Tayel MM, Al-Suwayeh SA. Ultra-fine self nanoemulsifying drug delivery system for transdermal delivery of meloxicam: Dependency on the type of surfactants. J Mol Liq. 2014;190:16-22.

Balakrishnan P, Lee B-J, Oh DH, Kim JO, Lee Y-I, Kim D-D, et al. Enhanced oral bioavailability of Coenzyme Q 10 by self-emulsifying drug delivery systems. Int J Pharm. 2009;374(1):66-72.

Balakumar K, Raghavan CV, Abdu S. Self nanoemulsifying drug delivery system (SNEDDS) of rosuvastatin calcium: design, formulation, bioavailability and pharmacokinetic evaluation. Colloids Surf B Biointerfaces. 2013;112:337-343.

Cerpnjak K, Zvonar A, Vrečer F, Gašperlin M. Development of a solid self-microemulsifying drug delivery system (SMEDDS) for solubility enhancement of naproxen. Drug Dev Ind Pharm. 2015;41(9):1548-57.

Constantinides PP, Chaubal MV, Shorr R. Advances in lipid nanodispersions for parenteral drug delivery and targeting. Adv Drug Deliv Rev. 2008;60(6):757-67.

Craig D, Barker S, Banning D, Booth S. An investigation into the mechanisms of self-emulsification using particle size analysis and low frequency dielectric spectroscopy. Int J Pharm. 1995;114(1):103-10.

Czajkowska-Kośnik A, Szekalska M, Amelian A, Szymańska E, Winnicka K. Development and evaluation of liquid and solid self-emulsifying drug delivery systems for atorvastatin. Molecules. 2015;20(12):21010-22.

Da Costa S, Basri M, Shamsudin N, Basri H. Stability of positively charged nanoemulsion formulation containing steroidal drug for effective transdermal application. J Chem. 2014;2014:748680.

Dash RN, Mohammed H, Humaira T, Ramesh D. Design, optimization and evaluation of glipizide solid selfnanoemulsifying drug delivery for enhanced solubility and dissolution. Saudi Pharm J. 2015;23(5):528-540. 
Divsalar A, Saboury AA, Nabiuni M, Zare Z, Kefayati ME, Seyedarabi A. Characterization and side effect analysis of a newly designed nanoemulsion targeting human serum albumin for drug delivery. Colloids Surf B Biointerfaces. 2012;98:80-4

Eid AM, El-Enshasy HA, Aziz R, Elmarzugi NA. The preparation and evaluation of self-nanoemulsifying systems containing Swietenia oil and an examination of its antiinflammatory effects. Int J Nanomedicine. 2014a;9:4685-95.

Eid AM, El-Enshasy HA, Aziz R, Elmarzugi NA. Preparation, characterization and anti-inflammatory activity of Swietenia macrophylla nanoemulgel. J Nanomed Nanotechnol. $2014 b ; 5: 190$.

Gao Z-G, Choi H-G, Shin H-J, Park K-M, Lim S-J, Hwang $\mathrm{K}-\mathrm{J}$, et al. Physicochemical characterization and evaluation of a microemulsion system for oral delivery of Cyclosporin A. Int J Pharm. 1998;161(1):75-86.

Gupta A, Eral HB, Hatton TA, Doyle PS. Nanoemulsions: formation, properties and applications. Soft Matter. 2016;12(11):2826-41.

Gutiérrez J, González C, Maestro A, Solè I, Pey C, Nolla J. Nano-emulsions: New applications and optimization of their preparation. Curr Opin Coll Int Sci. 2008;13(4):245-51.

Hecq J, Deleers M, Fanara D, Vranckx H, Amighi K. Preparation and characterization of nanocrystals for solubility and dissolution rate enhancement of nifedipine. Int J Pharm. 2005;299(1):167-77.

Hintzen F, Perera G, Hauptstein S, Müller C, Laffleur F, BernkopSchnürch A. In vivo evaluation of an oral self-microemulsifying drug delivery system (SMEDDS) for leuprorelin. Int J Pharm. 2014;472(1):20-6.

Kale AA, Patravale VB. Design and evaluation of selfemulsifying drug delivery systems (SEDDS) of nimodipine. AAPS PharmSciTech. 2008;9(1):191-6.

Keohane K, Rosa M, Coulter IS, Griffin BT. Enhanced colonic delivery of ciclosporin A self-emulsifying drug delivery system encapsulated in coated minispheres. Drug Dev Ind Pharm. 2016;42(2):245-53.

Klang V, Matsko NB, Valenta C, Hofer F. Electron microscopy of nanoemulsions: an essential tool for characterisation and stability assessment. Micron. 2012;43(2):85-103.
Kohli K, Chopra S, Dhar D, Arora S, Khar RK. Selfemulsifying drug delivery systems: an approach to enhance oral bioavailability. Drug Discov Today. 2010;15(21):958-65.

Kuentz M. Lipid-based formulations for oral delivery of lipophilic drugs. Drug Discov Today Technol. 2012;9(2):97-104.

Kumar CS. Nanotechnology tools in pharmaceutical R\&D. Mater Today 2010;12(Suppl 1):24-30.

Mahmoud DB, Shukr MH, Bendas ER. In vitro and in vivo evaluation of self-nanoemulsifying drug delivery systems of cilostazol for oral and parenteral administration. Int J Pharm. 2014;476(1):60-9.

Majeed H, Sharif HR, Haider J, Ali B, Fang Z. Preparation, characterization of Eugenol loaded nanoemulsions and their rheological characteristics Eur Acad Res. 2015;3(1):870-9.

Mancia G, Brown M, Castaigne A, de Leeuw P, Palmer CR, Rosenthal T, et al. Outcomes with nifedipine GITS or Co-amilozide in hypertensive diabetics and nondiabetics in Intervention as a Goal in Hypertension (INSIGHT). Hypertension. 2003;41(3):431-6.

Memvanga PB, Coco R, Préat V. An oral malaria therapy: Curcumin-loaded lipid-based drug delivery systems combined with $\beta$-arteether. J Control Release. 2013;172(3): 904-913.

Mohsin K, Rayan Alamri AA, Raish M, Alanazi FK, Hussain MD. Development of self-nanoemulsifying drug delivery systems for the enhancement of solubility and oral bioavailability of fenofibrate, a poorly water-soluble drug. Int J Nanomedicine. 2016;11:2829-38.

Nasr A, Gardouh A, Ghorab M. Novel solid self-nanoemulsifying drug delivery system (S-SNEDDS) for oral delivery of olmesartan medoxomil: design, formulation, pharmacokinetic and bioavailability evaluation. Pharmaceutics. 2016;8(3):20.

Nekkanti V, Karatgi P, Prabhu R, Pillai R. Solid selfmicroemulsifying formulation for candesartan cilexetil. AAPS PharmSciTech. 2010;11(1):9-17.

Nikalje AP. Nanotechnology and its applications in medicine. Med Chem. 2015;5(2):185-9.

Parveen S, Misra R, Sahoo SK. Nanoparticles: a boon to drug delivery, therapeutics, diagnostics and imaging. Nanomed Nanotech Biol Med. 2012;8(2):147-66. 
Pouton CW. Lipid formulations for oral administration of drugs: non-emulsifying, self-emulsifying and self-microemulsifying drug delivery systems. Eur J Pharm Sci. 2000;11(Suppl 2):93-8.

Pradhan S, Hedberg J, Blomberg E, Wold S, Wallinder IO. Effect of sonication on particle dispersion, administered dose and metal release of non-functionalized, non-inert metal nanoparticles. J Nanopart Res. 2016;18(9):285.

Richard S. Vascular effects of calcium channel antagonists: new evidence. Drugs. 2005;65(2):1-10.

Schuh RS, Bruxel F, Teixeira HF. Physicochemical properties of lecithin-based nanoemulsions obtained by spontaneous emulsification or high-pressure homogenization. Quim Nova. 2014;37(7):1193-98.

Shakeel F, Haq N, El-Badry M, Alanazi FK, Alsarra IA. Ultra fine super self-nanoemulsifying drug delivery system (SNEDDS) enhanced solubility and dissolution of indomethacin. J Mol Liq. 2013;180:89-94.
Stolnik S, Davies MC, Illum L, Davis SS, Boustta M, Vert M. The preparation of sub- $200 \mathrm{~nm}$ biodegradable colloidal particles from poly ( $\beta$-malic acid-co-benzyl malate) copolymers and their surface modification with Poloxamer and Poloxamine surfactants. J Control Release. 1994;30(1):57-67.

Swain S, Patra CN, Rao MB. Pharmaceutical drug delivery systems and vehicles. India: WPI Publishing; 2016.

Tadros T, Izquierdo P, Esquena J, Solans C. Formation and stability of nano-emulsions. Adv Colloid Interface Sci. 2004;108-109:303-318.

Vanani RN, Moezi L, Heli H. In vivo evaluation of a selfnanoemulsifying drug delivery system for curcumin. Biomed Pharmacother. 2017;88:715-20.

Received for publication on $17^{\text {th }}$ August 2017 Accepted for publication on $17^{\text {th }}$ August 2018 